\title{
A New Liposomal-Drug-in-Adhesive Patch for Transdermal Delivery of Sodium Diclofenac
}

\author{
Seyed Mojtaba Taghizadeh*, Sara Bajgholi
}

Novel Drug Delivery Systems Department, Polymer Science Faculty, Iran Polymer and Petrochemical Institute, Tehran, Iran. E-mail: ${ }^{*}$ S.M.Taghizadeh@ippi.ac.ir

Received October $16^{\text {th }}, 2011$; revised November $19^{\text {th }}, 2011$; accepted November $30^{\text {th }}, 2011$.

\begin{abstract}
Liposomes are known to have considerable potential as drug carriers such as liposomal suspension, freeze dried and cream-based systems among many other liposomal formulations. In this study a new drug-in-adhesive patch was fabricated using liposome-based nanocarrier. Transfersomes as ultra-deformable liposomes are based on phosphatidylcholin 95\% (phospholipon 90G) and phosphatidylcholin 50\% (phosal 50PG) were prepared and further optimized in a final acrylic patch system for effective adhesion. The prepared liposomes were added to an acrylic adhesive to obtain a new hybrid transdermal patch termed as "lipo-drug-in-adhesive" patch system. The sodium diclofenac was selected as a model drug and the permeation of the drug across rat skin was evaluated $(P>0.05)$, using the lipo-drug-in-adhesive patch system with various percentages of transfersomes $(4 \%-8 \% \mathrm{w} / \mathrm{w})$ and constant concentration of the drug (2\% $w / w)$. The peel strength and tack value of samples were also examined and quantified. The maximum flux of sodium diclofenac was observed in samples containing $8 \%(\mathrm{w} / \mathrm{w})$ phosphatidylcholin $50 \%$. The peel strength and tack value in samples containing phosphatidylcholin 50\% were lower than those samples containing phosphatidylcholin $95 \%$. It was observed that with increased amount of liposome in drug-in-adhesive patch system, the rate of skin permeation of the drug was also increased. It can be concluded that the developed lipo-drug-in-adhesive patch system enhances the drug release potential of transdermal delivering systems.
\end{abstract}

Keywords: Liposome, Sodium Diclofenac, Acrylic Adhesive, Peel Strength, Tack Value

\section{Introduction}

Many different methods are used for drug administration, such as oral, intravenous and intranasal ways. These routes are not free of disadvantages, for instance patient incompliance is the most serious limitation in intravenous administration. Oral applications may have some gastrointestinal (GI) adverse effects including peptic ulcer and gastrointestinal bleeding, and furthermore a significant fraction of drug may be eliminated by first-pass hepatic mechanism [1]. In this respect, transdermal drug delivery route is one of the most efficient systems for controlled drug administration, which eliminates all the disadvantages and causes no GI adverse effects and in general it is more patient compliant [2]. However penetration of drug across skin would be slow due to high barrier properties of skin. Therefore, advanced techniques using mixtures of chemical penetration enhancers in the form of microemulsions, micelles or liposomes have been developed to overcome drug administration inefficiencies over the recent decades [3,4]. Liposomes have been known for their considerable potential such as biocompatibility, non-immunogenicity and biodegradable properties as drug carriers. Due to their inherent structural specifities and properties liposomes are capable of encapsulating hydrophilic drugs inside their aqueous phase and hydrophobic drugs inside their phospholipids bilayers. Transfersomes which are elastic liposomes introduced by Cevc et al. can easily squeeze through the skin pores due to their more efficient permeation ability [5].

Liposomes can be applied transdermally in many compositional forms, such as suspension, freeze dried, cream and gel systems. Nevertheless the amount of released drug depends on the dose applied by patient in the form of cream or gel without any control in dose monitoring. The other compositional forms such as suspension and freeze dried liposomes require an additional preparation stage before any application [6,7].

Drug-in-adhesive patches have been used for transdermal drug delivery in recent years. These patches may 
offer benefits such as painless and easy use in delivery of therapeutic levels of drug, reduced dose frequency compared to the conventional oral dosage forms, and hardly any gastrointestinal effect being reported with repeated administrations [8].

In this study a new type of drug-in-adhesive patch was fabricated using liposomal-based nanocarrier to encapsulate or associate sodium diclofenac as a non-steroidalinflammatory drug. The non-functional acrylic pressure sensitive adhesive, Duro-Tak 4098, composed of acrylate-vinylacetate [9], have been used in this study to fabricate a new patch. The role of liposomes on drug release characteristics of transdermal drug-in-adhesive patch in relation to the peel strength and tack value of the whole acrylic adhesive patch is studied and reported.

\section{Materials and Methods}

\subsection{Materials}

Acrylic adhesive Duro-Tak ${ }^{\circledR} 87$ - 4098 was purchased from National Starch and Chemical, USA; CoTran 9720 as a release liner, and Scotchpak 1022 as a backing layer with thickness of $85 \mu \mathrm{m}$, were obtained from 3M, USA. Sodium cholate was purchased from Sigma, USA. Phospholipon $^{\circledR}$ 90G and Phosal ${ }^{\circledR} 50$ PG were kindly donated by Phospholipid, Germany. Chloroform for high performance liquid chromatography HPLC was purchased from Acros, UK. Methanol for liquid chromatography, and ethanol for analysis, orthophosphoric acid 85\% for analysis, di-potassium hydrogen phosphate anhydrous for analysis were purchased from Merck, Germany. Sodium diclofenac was kindly donated by Darou Pakhsh. Chem. Co. Iran.

\subsection{Preparation of Transfersomes}

Six elastic liposomal formulations of sodium diclofenac with compositions shown in Table $\mathbf{1}$ were prepared. Phospholipid and surfactant were taken in a clean, dry round-bottom flask and dissolved in solvent mixture of chloroform/methanol (2:1). Organic solvents were removed by a Buchi rotary evaporation (R-3000, Germany) above the lipid transition temperature. The remaining solvent was removed under vacuum overnight. The deposited lipid film was hydrated for $1 \mathrm{~h}$ with the drug solution in ethanol by rotating at $60 \mathrm{rpm}$ at $40^{\circ} \mathrm{C} \pm 1.0^{\circ} \mathrm{C}$ using a Unimax 1010 DT shaker incubator (Heizmodul, Heidolph, Germany). To prepare smaller liposomal vesicles, large multi-lamellar vesicles (LMLV)s were probe sonicated (Bandelin Electronic Sonopuls $\mathrm{GmbH} \&$ Co.KG, Germany) at $4^{\circ} \mathrm{C}$ for $20 \mathrm{~min}$ at $40 \mathrm{~W}$ [10].

\subsection{Characterization of Elastic Liposomal Formulations}

The prepared vesicles (LMLVs) were sonicated in a
Table 1. Material compositions of transfersomes based on phosphatidylcholin 95\%.

\begin{tabular}{cccc}
\hline \multirow{2}{*}{ Ingredients } & \multicolumn{3}{c}{ Formulations } \\
\cline { 2 - 4 } & $\mathrm{F}_{1}$ & $\mathrm{~F}_{2}$ & $\mathrm{~F}_{3}$ \\
\hline Sodium diclofenac $(\mathrm{mg})$ & 120 & 120 & 120 \\
Sodium cholate $(\mathrm{mg})$ & 28.8 & 43.2 & 57.6 \\
${ }^{(*)}$ Phosphatidylcholin 95\% $(\mathrm{mg})$ & 211.2 & 316.8 & 422.4 \\
Ethanol (mL) & 3 & 3 & 3 \\
\hline
\end{tabular}

${ }^{(*)} \mathrm{F}_{4}, \mathrm{~F}_{5}, \mathrm{~F}_{6}$ formulations were made with Phosphatidylcholin $50 \%$.

probe sonicator (Bandelin $\mathrm{GmbH}$ \& Co. KG, Germany) and the particle size of the vesicles before sonication was measured by a Jenavert optical microscope (Carl Zeiss, USA) shown in Figure 1. The smaller particle size of the probe sonicated vesicles were measured by dynamic light scattering (DLS) (Sematech-Goniometer SEM 633, France). Morphology of vesicles was obtained by TEM (Philips 400, KV80) as shown in Figure 2.

\subsection{Preparation of Lipo-Drug-in-Adhesive Patches}

The prepared solution of Table 1 was added into an acrylic adhesive and was stirred over night using rotary mixer. The resulting six elastic liposomal formulations of sodium diclofenac lipodrug-in-adhesive patches (LDIAPS)s were prepared with compositions shown as in Table 2. This mixture was spread over the backing layer using film applicator (Elcometer 3580) in order to obtain patches with uniform thickness. For removing the solvent, the coated films were kept in oven for $4 \mathrm{~h}$ at $32^{\circ} \mathrm{C}$ to dry and their thicknesses were measured by micrometer (mitutoyo 156 - 101, Mitutoyo, Japan) with the data presented in Table 3.

\subsection{Preparation of Rat Skin}

Male Sprague-Dawley rats (150 - $170 \mathrm{~g})$ obtained from Razi the Vaccine \& Serum Research Institute. At first, animals were sacrificed by excessive chloroform inhalation. The rodent hair was carefully removed with subsequent removal of subcutaneous fat layer with a scalpel. The prepared skin was then washed with distilled water and dried with the sterile gauze. The skin was wrapped in an aluminum foil and kept at $-30^{\circ} \mathrm{C}[11,12]$.

Permission of the experiment was approved by the Ethics Committee of the Faculty of Veterinary, Tehran Medical University.

\subsection{In Vitro Skin Permeation}

Permeation investigation was carried out using a completely removed rat abdominal skin in a well-characte- 


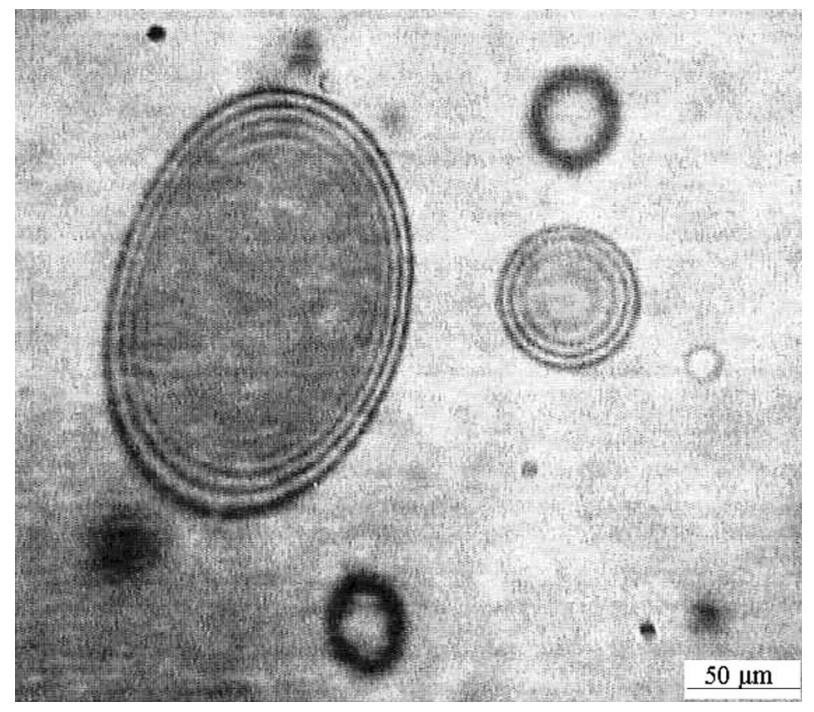

Figure 1. Vesicles' morphology before sonication was obtained by optical microscope.

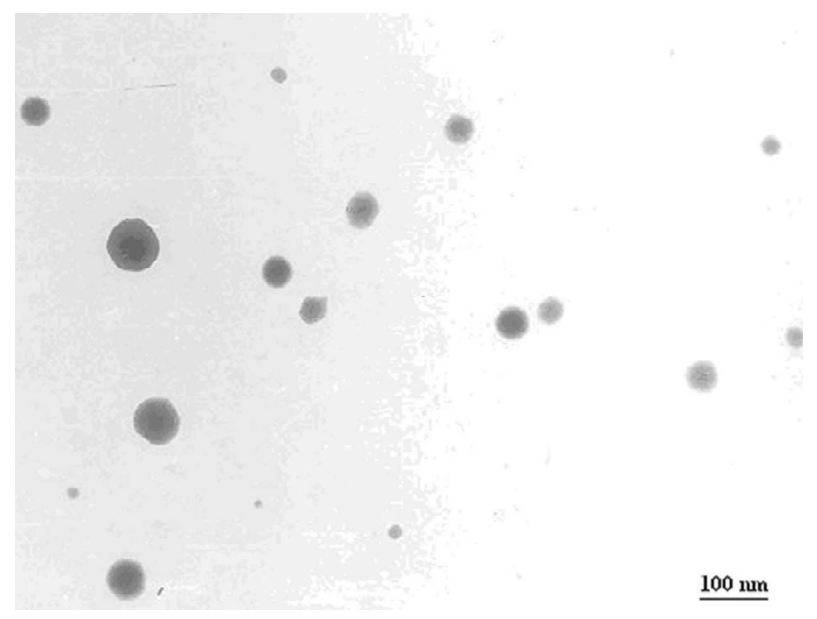

Figure 2. Vesicles' morphology was obtained by TEM.

Table 2. Material composition of patches based on phosphatidylcholin $95 \%$.

\begin{tabular}{cccc}
\hline & \multicolumn{3}{c}{ Formulations } \\
\cline { 2 - 4 } Ingredients & $\mathrm{F}_{1 \mathrm{P}}$ & $\mathrm{F}_{2 \mathrm{P}}$ & $\mathrm{F}_{3 \mathrm{P}}$ \\
\hline Sodium diclofenac (mg) & 120 & 120 & 120 \\
Sodium cholate (mg) & 28.8 & 43.2 & 57.6 \\
(*) $^{*}$ Phosphatidylcholin 95\% (mg) & 211.2 & 316.8 & 422.4 \\
Ethanol (mL) & 3 & 3 & 3 \\
Durotack 87- 4098 (mg) & 13430 & 13140 & 12850 \\
\hline
\end{tabular}

${ }^{(*)} \mathrm{F}_{4 \mathrm{P}}, \mathrm{F}_{5 \mathrm{P}}, \mathrm{F}_{6 \mathrm{P}}$ formulations were made with Phosphatidylcholin $50 \%$.

rized Chien permeation system with an effective diffusion area of $1 \mathrm{~cm}^{2}$ at $37^{\circ} \mathrm{C}$. Receptor compartment of dif-
Table 3. Dried thickness based of obtained patches (LDIAPSs).

\begin{tabular}{lcccccc}
\hline \multicolumn{7}{c}{ Formulations } \\
\hline \multirow{2}{*}{ Thickness $(\mu \mathrm{m})$} & $\mathrm{F}_{1 \mathrm{P}}$ & $\mathrm{F}_{2 \mathrm{P}}$ & $\mathrm{F}_{3 \mathrm{P}}$ & $\mathrm{F}_{4 \mathrm{P}}$ & $\mathrm{F}_{5 \mathrm{P}}$ & $\mathrm{F}_{6 \mathrm{P}}$ \\
& 100 & 100 & 100 & 101 & 99 & 99 \\
\hline
\end{tabular}

fusion cell was completely filled with $3 \mathrm{~mL}$ of phosphate buffer solution (PBS) with the $\mathrm{pH} 7.4$ as receiver medium. The rectangular film of the sample patch $\left(2.25 \mathrm{~cm}^{2}\right)$ was applied to the epidermal side of the rat skin with slight pressure and then mounted over the receptor compartment containing a magnetic stirrer. At predetermined time intervals $(0.5,1.5,3,5,8,24,48$ and $72 \mathrm{~h})$ the receptor medium was completely withdrawn from the receptor compartment and was replaced with fresh PBS. The sample solutions were analyzed for sodium diclofenac by UV absorbance at $276 \mathrm{~nm}$ in $0.1 \mathrm{M}$ phosphate buffer, $\mathrm{pH} 7.4$ and methanol. The standard curve was plotted in a concentration range of $1-36 \mu \mathrm{g} / \mathrm{mL}$ and $\mathrm{R}^{2}$ value was found to be 0.999 [13].

The permeation profiles of sodium diclofenac are shown in Figure 3 and the lag time and steady state flux is presented in Table $4(\mathrm{P}>0.05)$.

\subsection{Statistical Analysis}

The results were represented as mean $\pm \mathrm{SD}$ and the statistical Analysis was carried out by analysis of variance (ANOVA) at the 0.05 significance level.

\subsection{Probe-Tack Test}

Tack tests were carried out for adhesive tapes according to ASTM D3121 using a Chemie Instruments ProbeTack PT-500 (Fairfield, Ohio, USA) on at least five samples.

\subsection{Peel Strength Test at $180^{\circ}$}

Peel tests were carried out according to ASTM D3330 on adhesive coated tapes each with $25 \mathrm{~mm}$ width. After preparation of lipo-drug-in-adhesive patches consisting of the acrylic pressure sensitive adhesive tape/stainless steel joints, the samples were stored at room temperature for $20 \mathrm{~min}$. Peel force in 180 direction was measured at a peel rate of $30.50 \mathrm{~cm} / \mathrm{min}$ at room temperature using a Chemie Instruments adhesive/release tester AR-1000 (Fairfield). Peel tests were carried out on at least three samples of each adhesive tape using steel joint $[14,15]$.

\section{Results and Discussion}

According to study of Verma et al. particle size of liposome plays an important role in penetration of drugs into skin. The smaller liposome can easily pass through the 


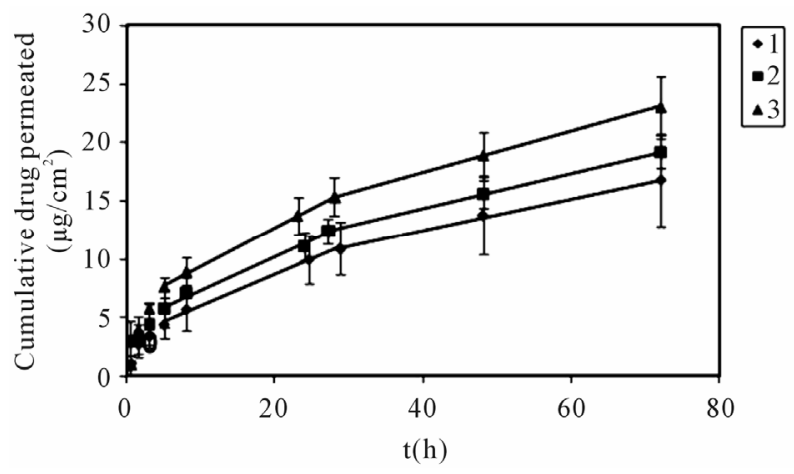

(a)

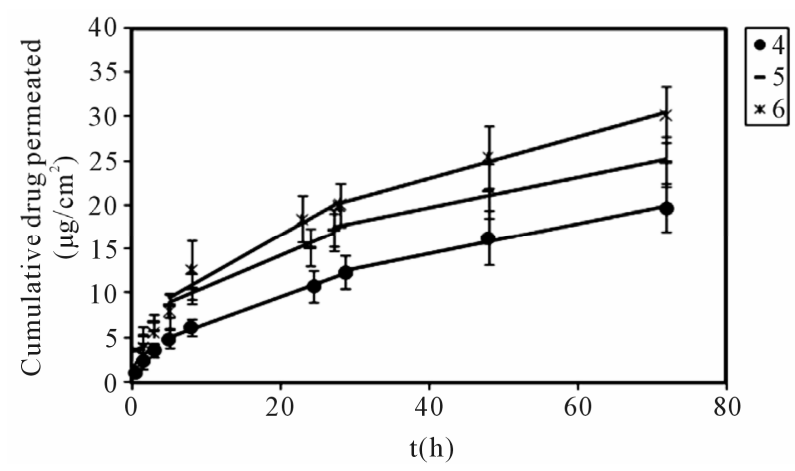

(b)

Figure 3. Cumulative sodium diclofenac permeated through excised abdominal rat skin versus time. (a) $F_{1 P}, F_{2 P}, F_{3 P} ;$ (b) $\mathbf{F}_{4 \mathrm{P}}, \mathbf{F}_{5 \mathrm{P}}, \mathbf{F}_{6 \mathrm{P}}$.

Table 4. Lag time and steady-state flux of sodium diclofenac through excised rat from lipo-drug-in adhesive patches.

\begin{tabular}{cccc}
\hline \multirow{2}{*}{ Formulations } & Lag time $(\mathrm{h})$ & \multicolumn{2}{c}{$\mathrm{J}_{\mathrm{ss}}\left(\mu \mathrm{g} / \mathrm{cm}^{2} \mathrm{~h}\right) \pm \mathrm{SD}$} \\
\cline { 3 - 4 } & & $(1-24 \mathrm{~h})$ & $(24-72 \mathrm{~h})$ \\
\hline $\mathrm{F}_{1 \mathrm{P}}$ & 0.67 & $0.267 \pm 0.04$ & $0.135 \pm 0.04$ \\
$\mathrm{~F}_{2 \mathrm{P}}$ & 0.34 & $0.283 \pm 0.05$ & $0.150 \pm 0.01$ \\
$\mathrm{~F}_{3 \mathrm{p}}$ & 0.09 & $0.331 \pm 0.04$ & $0.176 \pm 0.03$ \\
$\mathrm{~F}_{4 \mathrm{P}}$ & 0.56 & $0.304 \pm 0.04$ & $0.169 \pm 0.02$ \\
$\mathrm{~F}_{5 \mathrm{P}}$ & 0.27 & $0.350 \pm 0.04$ & $0.172 \pm 0.03$ \\
$\mathrm{~F}_{6 \mathrm{P}}$ & 0.06 & $0.477 \pm 0.11$ & $0.231 \pm 0.02$ \\
\hline
\end{tabular}

skin, whereas liposomes of larger sizes penetrate into the deeper layers of the skin with difficulty [16].

The mean size values of sodium diclofenac containing vesicles measured by dynamic light scattering. The particle size of transfersome based on phosphatidylcholin $50 \%$ and phosphatidylcholin $90 \%$ was $84.7 \pm 0.5 \mathrm{~nm}$ and $72.2 \pm 0.8 \mathrm{~nm}$ respectively.

The data show that liposomes based on phosphatidylcholin $50 \%$, of lower amount of phospholipid, are smaller than phosphatidylcholin $95 \%$ with higher amount of phospholipid. Following the addition of transfersome into acrylic pressure sensitive adhesives the microscopic observations indicated that the dispersion of transfersome in all the samples was uniformly distributed as in Figure 4.

The surfactant used in this study was sodium cholate. It was observed that the presence of this surfactant may assist transfersome particles pass through the skin pores of stratum corneum, significantly lower than the vesicle size, because sodium cholate renders flexibility to the bilayer lipid membranes of transfersomes According to Boinpally et al. by increasing the amount of sodium cholate in formulation, it is observed that lag time, which obtained by extrapolation, is being decreased [17].

In the $\mathrm{F}_{4 \mathrm{P}}, \mathrm{F}_{5 \mathrm{P}}, \mathrm{F}_{6 \mathrm{P}}$ phosphatidylcholin $50 \%$ may act as chemical penetration enhancer due to the presence of ethanol $(1.5 \%-2.5 \% \mathrm{w} / \mathrm{w})$ and propylene glycol $(33.8 \%$ $-41.2 \% \mathrm{w} / \mathrm{w})$ in the composition. As a result it would be expected that all formulations containing phosphatidylcholin $50 \%$ demonstrate maximum flux of sodium dicofenac [18].

The plots of cumulative amount of sodium diclofenac released across rat skin from different formulations as a function of time are given in Figure 3. Maximum flux of sodium diclofenac was $0.477 \pm 0.11 \mu \mathrm{g} / \mathrm{h} . \mathrm{cm}^{2}$ from the lipo-drug-in-adhesive patch system containing $8 \%(\mathrm{w} / \mathrm{w})$ liposome, based on phosphatidylcholin $50 \%, 2 \%(\mathrm{w} / \mathrm{w})$ sodium diclofenac and $90 \%(\mathrm{w} / \mathrm{w})$ acrylic adhesive. The range of flux obtained from phospholipid 50\% formulations is given in Table 4.

According to published reports $[19,20]$ by enhancing effects of liposomes of higher percentage the mean cumulative amounts of sodium diclofenac release increase across the rat skin (Figures 3(a) and 3(b)). Based on the cumulative amount of sodium diclofenac released during $72 \mathrm{~h}$, the study indicates that the order of sodium diclofenac passage across the epiderm is: $\mathrm{F}_{1 \mathrm{P}}<\mathrm{F}_{2 \mathrm{P}}<\mathrm{F}_{3 \mathrm{P}}<$ $\mathrm{F}_{4 \mathrm{P}}<\mathrm{F}_{5 \mathrm{P}}<\mathrm{F}_{6 \mathrm{P}}$.

As shown in Figure 5, the slopes between the time intervals of $1-28 \mathrm{~h}$ are sharper in comparison with the

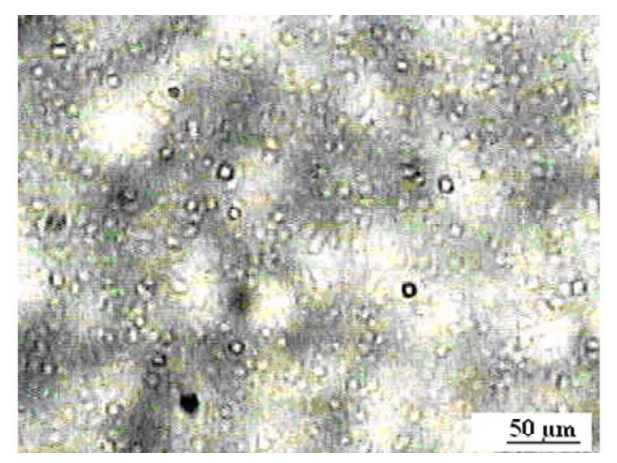

Figure 4. Dispersion of transfersomes in adhesive. 


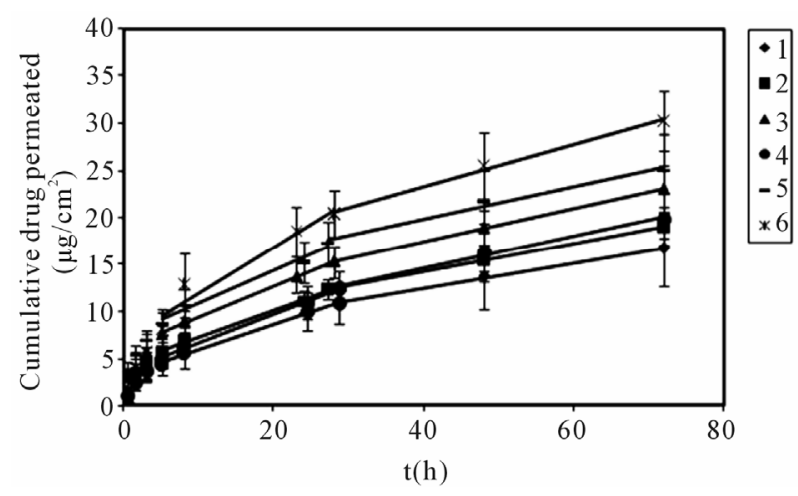

Figure 5. Cumulative sodium diclofenac permeated through excised abdominal rat skin versus time.

time intervals of $28-72 \mathrm{~h}$. From the shapes of the curves it may be concluded that during the first 28 hours the drug release takes place mainly from the surface, beyond which it is released from the bulk of the device. Figure 5 indicates that the drug release of the lipo-drug-in-adhesive patch system from the sample series of $F_{4 P}, F_{5 P}, F_{6 P}$ (phospholipon 50\%) was higher than the series of $\mathrm{F}_{1 \mathrm{P}}, \mathrm{F}_{2 \mathrm{P}}$, $\mathrm{F}_{3 \mathrm{P}}$ ( phospholipon 95\%) which were due to the smaller size parameter of former type of liposome and presence of propylene glycol and ethanol as penetration enhancers.

Nevertheless, the addition of any chemical penetration enhancer into the patch adhesive formulation would lower the adhesion properties, because of the plasticizing effect of the chemical penetration enhancers.

As shown in Table 5 by increasing the percentage of liposome and lower adhesive concentration at the contact surface, the adhesion properties of the lipo-drug-in-adhesive patch system were decreased in samples containing phosphatidylcholin $50 \%$ relative to phosphatidylcho$\operatorname{lin} 95 \%$.

\section{Conclusions}

Because of the general application of sodium diclofenac in treating rheumatic disorders, in this study we tried to formulate transdermal sodium diclofenac delivery systems with the novel design of having lipo-drug-in-adhesive patches using transfersomal liposome as penetration enhancer. The deformable liposome was prepared by surfactant such as sodium cholate to enhance its easy passage through the skin. It was observed that the flexible liposome in ethanol medium was compatible with the non-functional acrylic adhesive. The release of sodium diclofenac from the whole system across rat skin was evaluated. Finally it was confirmed that, the release of the drug in patches based on liposomal component such as phosphatidylcholin $50 \%$ is higher than phosphatidylcholin $95 \%$.
Table 5. Adhesion properties of lipo-drug-in adhesive patches.

\begin{tabular}{ccc}
\hline Formulation & Peel strength $(\mathrm{N} / 25 \mathrm{~mm})$ & Tack $\left(\mathrm{N} / \mathrm{mm}^{2}\right)$ \\
\hline $\mathrm{F}_{1 \mathrm{P}}$ & 4.7 & 4.64 \\
$\mathrm{~F}_{2 \mathrm{p}}$ & 4.62 & 4.58 \\
$\mathrm{~F}_{3 \mathrm{p}}$ & 3.54 & 2.53 \\
$\mathrm{~F}_{4 \mathrm{P}}$ & 3.42 & 3.71 \\
$\mathrm{~F}_{5 \mathrm{P}}$ & 3.21 & 3.36 \\
$\mathrm{~F}_{6 \mathrm{P}}$ & 3.05 & 2.39 \\
\hline
\end{tabular}

\section{Acknowledgements}

We are very thankful to Ms. Houri Mivehchi for editing the manuscript.

\section{Declaration of Interest}

Support of this work from Iran Polymer and Petrochemical Institute is appreciated.

\section{REFERENCES}

[1] K. B. Ita, J. D. Preez, M. E. Lane, et al., "Dermal Delivery of Selected Hydrophilic Drugs from Elastic Liposomes: Effect of phospholipid Formulation and Surfactants," Journal of Pharmacy and Pharmacology, Vol. 59, No. 9, 2007, pp. 1215-1222. doi:10.1211/jpp.59.9.0005

[2] M. Manconi, S. Mura, C. Sinico, et al., "Development and Characterization of Liposomes Containing Glycols as Carriers for Diclofenac," Colloids and Surfaces A: Physicochemical and Engineering Aspects, Vol. 342, No. 1-3, 2009, pp. 53-58. doi:10.1016/j.colsurfa.2009.04.006

[3] L. B. Lopes, M. V. Scarpa and G. V. J. Silva, "Studies on the Encapsulation of Diclofenac in Small Unilamellar Liposomes of Soya Phosphatidylcholin," Colloids and Surfaces B: Biointerfaces, Vol. 39, No. 4, 2004, pp. 151-158. doi:10.1016/j.colsurfb.2004.09.004

[4] P. Karande and S. Mitragotri, "Enhancement of Transdermal Drug Delivery via Synergistic Action of Chemicals," Biochimica et Biophysica Acta (BBA)-Biomembranes, Vol. 1788, No. 11, 2009, pp. 2362-2373. doi:10.1016/j.bbamem.2009.08.015

[5] M. M. A. Elsayed, O. Y. Abdallah, V. F. Naggar, et al., "Lipid Vesicles for Skin Delivery of Drugs: Reviewing Three Decades of Research," International Journal of Pharmaceutics, Vol. 332, No. 1-2, 2007, pp. 1-16. doi:10.1016/j.ijpharm.2006.12.005

[6] J. A. Zhang, G. Anyarambhatla, L. Ma, et al., "Development and Characterization of a Novel Cremophor EL Free Liposome-Based Paclitaxel (LEP-ETU) Formulation," European Journal of Pharmaceutics and Biopharmaceutics, Vol. 59, No. 1, 2005, pp. 177-187. doi:10.1016/i.ejpb.2004.06.009

[7] K. Shimizu, M. Osada, K. Takemoto, et al., "Tempera- 
ture-Dependent Transfer of Amphotericin B from Liposomal Membrane of Ambiosome to Fungal Cell Membrane," Journal of Controlled Release, Vol. 141, No. 2, 2010, pp. 208-215. doi:10.1016/i.jconrel.2009.09.019

[8] C. Ren, L. Fang, L. Ling, et al., "Designand in Vivo Evaluation of an Indapamide Transdermal Patch," International Journal of Pharmaceutics, Vol. 370, No. 1-2, 2009, pp. 129-135. doi:10.1016/j.ijpharm.2008.12.004

[9] E. Gutschke, S. Bracht, S. Nagel, et al., "Adhesion Testing of Transdermal Matrix Patches with a Probe Tack Test: In Vitro and in Vivo Evaluation," European Journal of Pharmaceutics and Biopharmaceutics, Vol. 75, No. 3, 2009, pp. 399-404. doi:10.1016/j.ejpb.2010.03.016

[10] T. Garg, S. Jain, S. H. Pal, et al., "Elastic liposomal Formulation for Sustained Delivery of Antimigraine Drugs: In Vitro Characterization and Biological Evaluation," Drug Development and Industrial Pharmacy, Vol. 34, No. 10, 2008, pp. 1100-1110. doi:10.1080/03639040801965079

[11] A. Mehdizadeh, M. H. Ghahremani, M. R. Rouini, et al., "Effects of Pressure Sensitive Adhesives and Chemical Permeation Enhancers on the Permeability of Fentanyl through Excised Rat Skin," Acta Pharmaceutica, Vol. 56, No. 2, 2006, pp. 219-229.

[12] B. Vora, A. J. Khopade and N. K. Jain, "Proniosome Based Transdermal Delivery of Levonorgestrel for Effective Contraception," Journal of Controlled Release, Vol. 54, No. 2, 1998, pp. 149-165. doi:10.1016/S0168-3659(97)00100-4

[13] M. Kincl, M. Meleh, M. Veber, et al., "Study of Physicochemical Parameters Affecting the Release of Diclofenac Sodium from Lipophilic Matrix Tablets," Acta Chimica Slovenica, Vol. 51, 2004, pp. 409-425.
[14] S. M. Taghizade, A. Soroushnia and F. Mohamadnia, "Preparation and in-Vitro Evaluation of a New Fentanyl Patch Based on Functional and Non-Functional Pressure Sensitive Adhesives," AAPS PharmSciTech, Vol. 11, No. 1, 2010, pp. 278-284. doi:10.1208/s12249-009-9366-3

[15] S. M. Taghizadeh and D. Ghasemi, "Synthesis and Optimization of a Four-Component Acrylic-Based Copolymer as Pressure Sensitive Adhesive," Iranian Polymer Journal, Vol. 19, No. 5, 2010, pp. 343-352.

[16] D. D. Verma, S. Verma, G. Blume, et al., "Particle Size of Liposomes Influences Dermal Delivery of Substances into Skin," International Journal of Pharmaceutics, Vol. 258, No. 1-2, 2003, pp. 141-151. doi:10.1016/S0378-5173(03)00183-2

[17] R. R. Boinpally, S. L. Zhou, S. Poondru, et al., "Lecithin Vesicles for Topical Delivery of Diclofenac," European Journal of Pharmaceutics and Biopharmaceutics, Vol. 56, No. 3, 2003, pp. 389-392. doi:10.1016/S0939-6411(03)00143-7

[18] B. EI-Houssieny and H. M. Hamouda, "Formulation and Evaluation of Clotrimazole from Pluronic $F_{127}$ Gels," Drug Discoveries \& Therapeutics, Vol. 4, 2010, pp. 3343.

[19] D. Dhamecha, A. A. Rathi and M. Saifee, "Drug Vehicle Based Approaches of Penetration Enhancement," International Journal of Pharmacy and Pharmaceutical Sciences, Vol. 1, No. 1, 2009, pp. 24-46.

[20] R. K. Bhardwaj and T. Velpandian, "Effect of Liposomes on Permeation of Diclofenac through Cadaver Skin: In-Vivo Evaluation Using Animal Models," Journal of Pharmacy and Pharmacology, Vol. 6, No. 11, 2010, pp. 485-489. 